\title{
WORK STRUCTURING FOR FLOW
}

\author{
Nelly P. Garcia-Lopez ${ }^{1}$, Martin Fischer², and Luis F. Alarcón ${ }^{3}$
}

\begin{abstract}
Achieving smooth production flow has been one of the main objectives underlying lean manufacturing and construction. To achieve production flow, field managers rely on work structuring methods to enable them to structure activities and flows. Current work structuring methods enable field managers to structure activities, but they do not explicitly represent all seven construction flows or their movement through the project. Hence, field managers rely on their intuition and tacit understanding of flow sequencing, which can cause communication problems between stakeholders resulting in delays and productivity loss. This paper presents a work structuring method that allows field managers to explicitly represent construction activities, flows, and flow movement through the project. The work structuring method was tested prospectively at three construction sites with different scopes and planning methods. The work structuring method allows field managers to generate activity and flow-based schedules to plan and control the project. Furthermore, it improves stakeholder understanding of the plan by visually representing activities' and flows' interdependencies.
\end{abstract}

\section{KEYWORDS}

Work flow, work structuring, flow integration, production management, lean construction.

\section{INTRODUCTION}

Managing construction projects to achieve flow has been one of the ideas advocated by Lean production to deliver projects maximizing value and minimizing waste. Optimal production flow entails synchronizing the flow of operations, processes, and projects (Sacks 2016). Within a project, production flow is achieved when all flows needed to execute an activity are available at the right time and in the correct amounts for activities to be executed efficiently (Bertelsen et al. 2007). Good field managers actively think about construction flows when they are creating a plan. It is common for field managers to mention concepts such as planning the handoffs between trades, keeping the rhythm or pace, and feeding the activities (Garcia-Lopez 2017). Field managers rely on work structuring methods to answer these questions and ultimately enable them to generate

\footnotetext{
Project Director, Grupo Galopa, Bogotá, Colombia, +57 305297 7320, ngarcia@ grupogalopa.com

2 Kumagai Professor of Engineering and Professor of Civil and Environmental Engineering and Director, Center for Integrated Facility Engineering (CIFE), Stanford University, Stanford, CA, fischer@stanford.edu

3 Professor, Department of Construction Engineering and Management, Pontificia Universidad Católica de Chile, Santiago, Chile, +56 22354 7165, lalarcon@ing.puc.cl
} 
schedules where activities and flows feeding those activities are coordinated among project stakeholders. To do this, work structuring methods must enable field managers to define what activities are needed to execute the project, what flows are needed to execute the activities, and how activities and flows need to be sequenced. Koskela (1999) classified construction flows into seven types: labor, equipment, workspace, materials, precedence, information, and external flows. While current work structuring methods allow field managers to structure construction activities and some of the construction flows, they do not explicitly represent all seven construction flows and their movement through the project. Hence, field managers still rely on a tacit understanding of flow requirements and flow movement, which can lead to miscommunication about the plan among project stakeholders resulting in construction delays in the field. This paper presents a work structuring method that enables field managers to explicitly structure activities, all seven flows, and their sequencing.

\section{WORK STRUCTURING METHODS}

In lean construction, production system design, or 'work structuring', entails connecting the facility design (product) with the processes, typically in the form of schedules, used to deliver the physical facility (Ballard et al. 2001; Tsao et al. 2004). The objective of work structuring is to enable field managers to generate reliable schedules, using methods such as Last Planner, where flows are coordinated so that handoffs between production units are clear to all project stakeholders, and flows are balanced so that their availability is synchronized with activity demand while maximizing flow utilization.

There are currently two main methods for work structuring: Ballard's work structuring method that has been advanced by other Lean construction researchers (Ballard 1999; Ballard et al. 2001; Tsao et al. 2004), and Takt planning (Tommelein 2017). Both methods allow field managers to structure activities and focus on explicitly defining precedence, labor, and workspace flows. However, information, material, equipment, and external flows are not explicitly represented.

Through six steps Ballard's work structuring method focuses on activity definition, sequencing, and assignment: (1) breaking down work into units that can be assigned to specialists (activity definition), (2) sequencing activities, (3) understanding how work will be handed off between specialists, (4) understanding whether work will be executed continuously between locations, (5) placing and sizing decoupling buffers, and (6) scheduling activities (Tsao et al. 2004). Precedence and workspace flows are fully structured by determining the activity and workspace sequencing. Labor flows are partially structured by assigning work to a specific specialist (i.e., labor flow class) and understanding how the specialist moves between workspaces, but labor mobilizations (offsite flows) are not included. External, information, material, and equipment flows are not supported.

Takt planning is another method for work structuring (Frandson et al. 2013; Frandson and Tommelein 2014; Tommelein 2017). The objective of Takt planning is to set a pace, known as Takt time, at which each trade can complete its assigned units of work in a zone. Takt planning is carried out in five iterative steps: "(1) data gathering, (2) zone and Takt 
time definition, (3) trade sequence identification, (4) determination of individual trade durations, (5) workflow balancing, (6) production schedule finalization" (Frandson and Tommelein 2014). Takt planning allows field managers to structure on-site flows related to labor, equipment, workspace, and precedence flow types. However, it lacks support for structuring material, information, equipment and external flows.

Another difficulty faced by field managers during planning is developing schedules that can be used to communicate the plan and control work in the field. This problem arises because existing construction models used to represent construction work do not fully represent all construction flows, and their sequencing and movement through the project (Garcia-Lopez and Fischer 2016). Critical Path Method (CPM) schedules represent precedence flows, and line-of-balance schedules represent workspace and precedence flows (Kenley and Seppänen 2009). While both schedule representations can include resources as activity attributes, they do not explicitly represent flow sequencing. Similarly, neither of the schedule representations represents off-site flows, which are flows that originate outside of the site, such as material deliveries, resource mobilizations, or information requirements. Hence, field managers' flow planning knowledge cannot be formally embedded in construction schedules and remains tacit in planners' minds. This can result in communication problems between stakeholders, low planning reliability, and reduced productivity in the field. To help close this gap, the authors developed an activity and flow model, called the Activity-Flow Model (AFM), that allows field managers to formally represent, track, and control construction activities and flows (Garcia-Lopez 2017). The work structuring method subject of this paper depends on the AFM representation. Hence, we will first present a summary of the AFM followed by the development of the work structuring method.

\section{ACTIVITY-FLOW MODEL (AFM)}

The AFM is a construction model composed of a set of production planning, production control, and prediction methods for managing activities and flows. It is based on the Construction Physics conceptualization which extends the seven-flow conceptualization introduced by Koskela (1999) by suggesting that flows can be viewed as physical entities feeding activities (Bertelsen et al. 2007).

Figure 1 summarizes the conceptual activity and flow model underpinning the AFM. Construction activities are defined as resources acting on components (Darwiche et al. 1988; Fischer and Aalami 1996) that need a certain set of flows to be executed efficiently (Bertelsen et al. 2007). There are two mechanisms that can cause variation in the readiness of flows feeding activities: the occurrence of variability factors such as bad weather, and late release of flows due to delays in upstream activities (González et al. 2009). Buffers can be implemented by field mangers to shield activities from variation in the flows (González et al. 2011). If a flow's readiness variation is larger than its time buffer, the flow delays the activity's start. At any time, one or more of the flows needed by an activity can be experiencing variations. The activity's start is constrained by the flow with the highest variation, which is known as the critical flow, i.e., even if the other flows were ready the 
activity would still be unable to start due to the unavailability of the critical flow (Bertelsen et al. 2006).

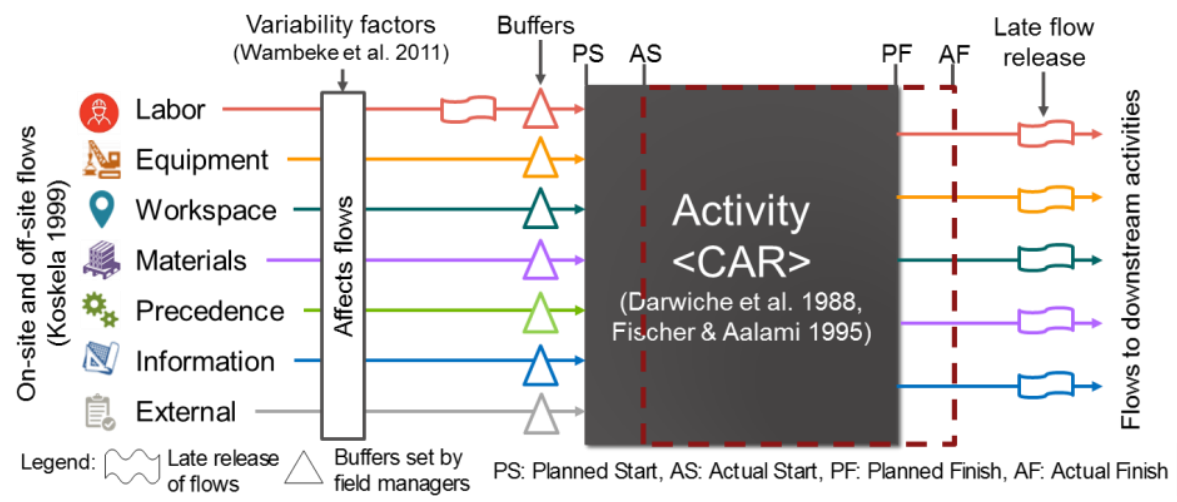

Figure 1: Conceptual activity and flow model showing a construction activity, the seven flows, and their variation mechanisms.

The AFM formalizes the conceptual activity and flow model in an ontology that was operationalized in a class diagram implemented in a web application. The AFM represents the schedule as a network of on-site flows joining the activities and off-site flows feeding the activities (Figure 2). During production control, field managers track the status of the activities and flows, which are used by the AFM to compute activity and flow variation metrics. The AFM leverages activity and flow data collected during production control to generate analytics and statistically significant predictions about the downstream activities that are most likely to face variations (Fischer et al. 2018). In test projects, these analytics and predictions have been used to allocate resources, size buffers, and modify the lookahead schedule aiming at improving schedule conformance (Garcia-Lopez 2017).

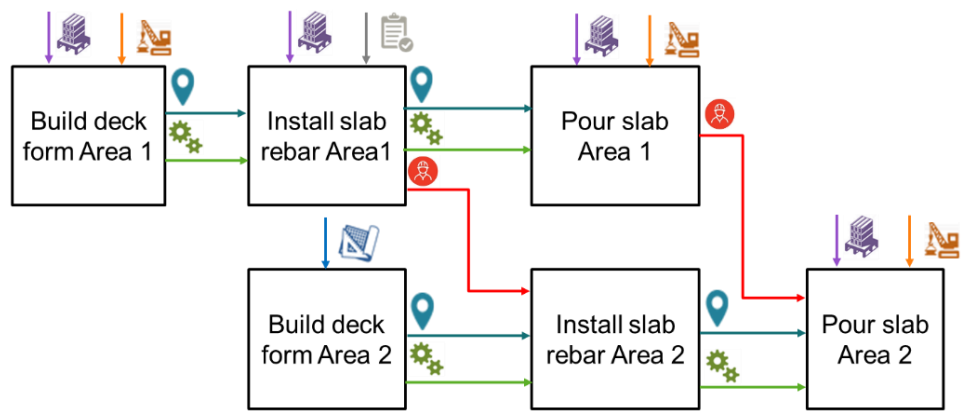

Figure 2: Example of schedule representation showing on-site flows joining the activities and off-site flows feeding the activities.

Activity and flow schedules can be generated and controlled using methods advocated by the Last Planner System (Hamzeh et al. 2012). The difference lies in the underlying schedule representation used by the AFM, which explicitly represents and tracks the status of both activities and flows. The AFM is initialized by inputting an activity and flow-based schedule that forms the basis for re-planning and controlling the project. Hence, field managers need work structuring methods that enable them to structure the project's activities, flows, and their sequencing to create activity and flow-based schedules. 


\section{ACTIVITY AND FLOW WORK STRUCTURING METHOD}

The Activity and Flow-based Work Structuring Method (AFWSM) allows field managers to structure the activities and flows in a construction project to generate activity and flow-based schedules. Construction schedules are composed of fragnets, which are sequences of activity types that are repeated in different workspaces in the project. Hence, field managers only need to structure a construction fragnet's activities and flows, and then replicate the logic embedded in the fragnet into the full schedule.

Fragnets are determined by the construction method chosen by field managers to build different components on a project (Dong 2012; Fischer and Aalami 1996). For example, building a slab (building component) can be accomplished by choosing the construction method cast in place slab or prefabricate slab. The choice of construction method determines the activity types and flows that are needed to execute on-site work.

We developed the AFWSM by reviewing existing literature to understand what flow information was needed by field managers to plan and control on-site work and carried out interviews to inquire how that information could be elicited from field managers and be formally represented in a plan. We validated the work structuring method prospectively by implementing it on three construction test sites with different scopes, planning methods, and control methods.

The AFWSM has seven steps encompassing activity definition, activity sequencing, flow definition, and flow sequencing: (1) choose a construction method and identify activity types for the fragnet, (2) sequence activity types based on precedence flows for the fragnet, (3) identify workspaces and their sequencing, (4) identify on-site flows, (5) identify off-site flows, (6) identify flows interfacing with other fragnets, (7) identify stakeholders responsible for the flows. These steps are carried out for each of the fragnets needed to execute the project and are shown graphically in Figure 3, using the case example for the shell construction from one of the test projects.

The first step is to choose a construction method and identify activity types for the fragnet. Activity types are defined as <Component, Action, Resource> tuples. The second step is to identify the sequencing of the activity types based on their precedence flows only. Precedence flows represent physical or technical constraints between the activities. Notice that steps one and two mirror the first two steps of Ballard's work structuring method, which determine the activity definition and activity sequencing. The third step is to identify the workspaces where each of the activity types in the fragnet will be executed and the sequencing for the workspaces. This step is similar to zone definition in Takt planning or location sequencing in the line-of-balance scheduling method. Tommelein (2017) proposes an excellent method for defining zones by balancing work quantities used by the different activities in a project. The fourth step is to identify the on-site flows that are required to execute each of the activity types for the fragnet and how they are released between the activity types. This step identifies additional activity relationships that are not captured by precedence flows identified in step two. This is achieved by asking field managers: Why does activity type $\mathrm{x}$ go after activity type $\mathrm{y}$ ? What does activity type $\mathrm{x}$ need from activity type $y$ ? The field manager's response is then classified into one or more of the seven types of flows. In the case example, the activity type "Install column forms" goes after the activity type "Install column rebar" because it occupies the same workspace (workspace 
flow - blue arrow), it encloses the rebar causing a precedence constraint (precedence flow - green arrow), and it works on the same column component (material flow - purple arrow). Generally, additional information needs to be asked regarding the labor flows to understand crew composition and how crews move between activity types and across workspaces. This is achieved by asking field managers: What crews execute each of the activity types? Is there more than one crew executing this activity type simultaneously in a different workspace? How do crews move between activity types (within the same workspace)? How do crews move between workspaces? The fifth step is to identify the off-site flows that are required to execute each of the activity types. This is achieved by asking field managers whether any information, external permits/inspections, materials, labor mobilizations, or equipment mobilizations are needed to execute each of the activity types.

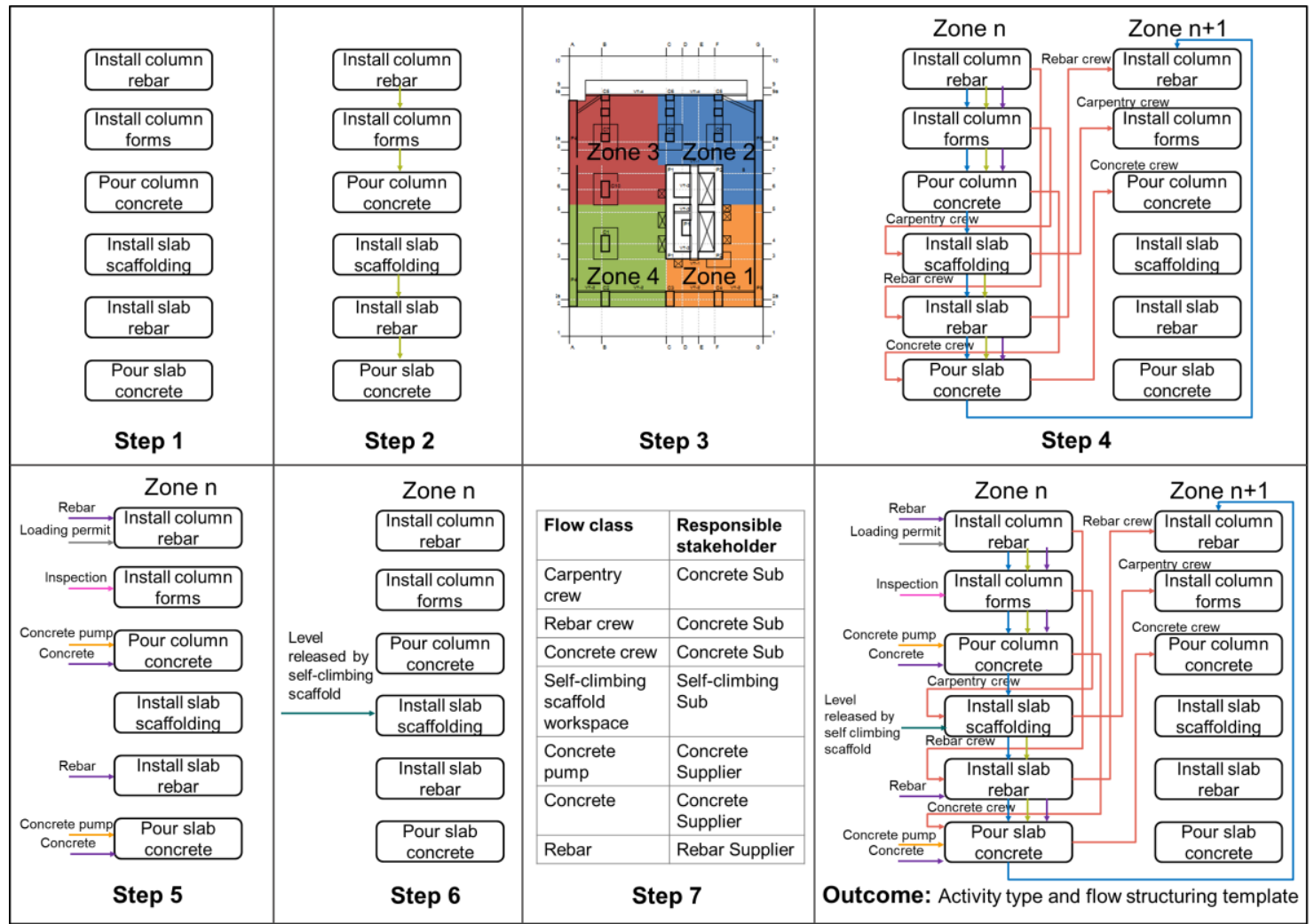

Figure 3: Figure showing an example of the steps of the AFWSM for a structural shell fragnet. The flows are color-coded as follows: labor in red, equipment in orange, workspace in blue, precedence in green, material in purple, information in pink, and external in gray.

The sixth step consists of identifying if any activity type needs a flow that originates from another fragnet. This allows the method to represent interdependencies between different fragnets in the project. In the case example, the activity type "Install slab scaffolding" requires the workspace occupied by the activity type "Raise self-climbing scaffold," which belongs to the elevator shaft fragnet. Step seven consists of asking field managers to identify the responsible stakeholder for each of the on-site and off-site flows. 
Normally, the responsible stakeholder for the flow is the subcontractor responsible for executing the activity type. However, sometimes the responsible stakeholder can be a supplier, a designer, or the GC.

The outcome of the AFWSM is a template of the prototypical activity types and flow representation for a fragnet. The diagram shows on-site flows joining the different activity types, off-site flows feeding the activity types, and flows interfacing with activity types belonging to other fragnets. Hence, it explicitly shows the interdependencies between activities and flows.

The activity types and flows in the AFWSM template represent a typical fragnet. Since project schedules are composed of fragnets executed at different workspaces, each activity in the project schedule can be mapped to an activity type contained in the fragnet's activity type and flow structuring template. Hence, field managers can create activity and flow schedules that can be used to plan, track, and control the project using the AFM.

\section{IMPLEMENTATION RESULTS}

The AFSWM should be able to represent a wide variety of construction methods, be used to transform different schedule representations into an activity and flow-based representation and require a low time commitment from the field manager's team. This section presents the validation results of applying the proposed work structuring method on three test projects that had different scopes and planning methods.

The first project was the Ichma office building located in Peru. We tracked this project during a total of 18 weeks during its structural phase, with the lead researcher spending the first 4 weeks on site, the following 10 weeks remotely, and the final 4 weeks on site. This project was extremely sophisticated in using state of the art planning and control methods. Field managers implemented the whole Last Planner System (master schedule, phase schedule, look-ahead, and weekly). Additionally, they carried out Takt planning at the phase schedule level to design their operations: choosing how to break down the workspaces depending on the quantities and balancing the resources based on historical productivity rates. They controlled the project by using look-ahead, weekly, and daily planning. Each activity was assigned a clearly delineated workspace. Each project engineer created a daily plan for their scope of work and tracked the daily execution against the plan, assigning daily reasons for variation to the activities.

The second project was the Equilibrium residential building located in Colombia. We tracked this project for a total of four weeks during the foundations phase (deep caissons). This project used traditional CPM scheduling to plan the project. The CPM schedule was developed at very high level of detail, containing activities not exceeding one week in duration. Field managers used a CPM schedule to control the project by updating the actual start and actual finish for the activities to have a historic record of the project progress and assess schedule slippages. Additionally, they implemented the Last Planner's weekly planning and control process involving the subcontractors in a collaborative way.

The third project was the Frederikskaj residential blocks located in Denmark. We tracked this project for a total of four weeks during the interior finishing phase. This project 
used line-of-balance scheduling to plan the project and a Location-based Management System to control the progress.

All the field managers in the test projects were interested in improving the planning and control methods used in their projects. They wanted to understand whether the AFWSM could help them to better coordinate and communicate the plan among the different project stakeholders.

In each of the test projects we first used the AFWSM to model the flow logic for each of the fragnets contained in their look-ahead schedule. This was a collaborative process with field managers, who explained the construction methods and checked the representations. Second, we used the results of the AFWS to transform the project's existing look-ahead schedule into an activity and flow schedule.

\section{AFWSM IMPLEMENTATION RESULTS}

In total, we used the AFWSM to model 11 fragnets spanning from caisson foundations to interior finishes (Table 1). In the Ichma project, we modelled 5 fragnets: elevator shaft construction, structural shell construction, core beams and slabs, interior floors, and interior walls. For the Equilibrium project, we modelled 3 fragnets: caissons, in-caisson walls and columns, and foundation beams. Finally, we modelled 3 fragnets for the Frederikskaj project: walls and in-wall MEP, floor construction, and interior finishes. This provides evidence of the generality of the AFWSM, since it was used to represent fragnets spanning three key phases of a construction project.

Table 1. Summary of the fragnets modelled using the AFWSM.

\begin{tabular}{cccccc}
\hline Project & Fragnet & $\begin{array}{c}\text { \# Activity } \\
\text { types }\end{array}$ & $\begin{array}{c}\text { \# } \\
\text { Flows }\end{array}$ & $\begin{array}{c}\text { \# Flows/ } \\
\text { \#Activity types }\end{array}$ & $\begin{array}{c}\text { Time } \\
\text { (mins) }\end{array}$ \\
\hline Ichma & Elevator shaft & 6 & 15 & 2.5 & 15 \\
(Takt) & Structural shell & 6 & 22 & 3.7 & 20 \\
& Core beams \& slabs & 7 & 23 & 3.3 & 20 \\
& Interior floors & 3 & 10 & 3.3 & 10 \\
& Walls \& in-wall MEP & 4 & 15 & 3.8 & 20 \\
\hline Equilibrium & Caissons & 4 & 7 & 1.8 & 10 \\
(CPM) & In-caisson walls \& & 3 & 10 & 3.3 & 10 \\
& columns & & & & 10 \\
\hline Frederikskaj & Foundation beams & 4 & 14 & 3.5 & 20 \\
(LOB) & Wall \& MEP & 6 & 13 & 2.2 & 15 \\
& Floor construction & 5 & 16 & 3.2 & 20 \\
\hline & Interior finishes & 17 & 51 & 3.0 & $\mathbf{1 5}$ \\
\hline
\end{tabular}

Another important requirement was related to the time commitment needed from field managers to implement the method. The average time required to model a fragnet was 15 minutes, the longest time was 20 minutes, and the shortest time was 10 minutes. We asked field managers how much time they would be willing to spend per week to apply the AFWSM on their projects and the average reply was thirty minutes. Hence, the amount of time that it takes to apply the AFSWM is acceptable. 


\section{DEVELOPMENT OF ACTIVITY AND FLOW-BASED SCHEDULES}

To develop activity and flow-based schedules, we transformed the four-week lookahead schedule developed by field managers into an activity-flow representation by using the results of the AFWSM (Table 2). The Ichma project represented its look-ahead schedule in a spreadsheet using a takt representation where the rows represented the activity types, the columns represented time (days), and the cells contained the workspaces where the work was executed. The Equilibrium project used a CPM representation. Finally, the Frederikskaj project used a line-of-balance representation.

On average, we spent 1.4 hours transforming a project's look-ahead schedule into an activity and flow-based representation after applying the AFWSM. As expected, the time it took to transform the schedule into an activity and flow-based representation depended on the number of activities and flows that were in the schedule. The look-ahead schedule for the Frederikskaj project contained the biggest number of activities (311) and took 2 hours to create. The Ichma project contained 238 activities and took 1.2 hours to create. Finally, the Equilibrium project contained the lowest number of activities (111) and took 1 hour to create. It is necessary to reduce the amount of time it takes to transform the activity-based look-ahead into an activity and flow-based look-ahead for the method to be used extensively on construction projects.

Table 2. Number of activities and flows in the project 4-week lookaheads and time needed to prepare them.

\begin{tabular}{|c|c|c|c|}
\hline Project & $\begin{array}{c}\text { \# Activities in } \\
\text { look-ahead plan (4 } \\
\text { weeks) }\end{array}$ & $\begin{array}{c}\text { \# Flows in } \\
\text { look-ahead } \\
\text { plan }\end{array}$ & $\begin{array}{c}\text { Time } \\
\text { (hours) }\end{array}$ \\
\hline Ichma (Takt) & 238 & 1,002 & 1.2 \\
\hline Equilibrium (CPM) & 111 & 442 & 1.0 \\
\hline Frederikskaj (LOB) & 311 & 1,210 & 2.0 \\
\hline Average & $\mathbf{2 2 0 . 0}$ & $\mathbf{8 8 4 . 7}$ & $\mathbf{1 . 4}$ \\
\hline
\end{tabular}

Overall, field managers valued how the AFSWM allowed them to formally map and structure construction flows: "I think it's very useful that we have a tool that formally maps the flows that are needed to execute an activity that is in the plan ... These things pass through our heads, but there is no formal tool that allows us to check that all the flows are ready so the activity is not in danger" (Project engineer, Ichma). Additionally, they highlighted how the AFWSM allowed them to communicate the plan visually between stakeholders, especially regarding the movement of labor flows between activities and workspace handoffs, leading to improvements in project coordination and plan understanding.

\section{CONCLUSIONS AND FUTURE WORK}

The AFWSM extends current work structuring methods by allowing field managers to structure activity and flow sequencing for each of the fragnets on a project. The activity type and flow structuring template enables work structuring communication between project stakeholders and the understanding of the interfaces between the different activity 
types and flows. The AFWSM allows field managers to transform existing activity-based schedules into an activity and flow-based representation that can be used to plan and control the project using the AFM.

Future research is needed to evaluate the impact of the use of the AFWSM on project performance both by collecting qualitative evaluations from users and measuring quantitative impacts through case studies.

An important next step in this research is improving the time it takes to extend existing schedules into an activity and flow representation. A potential research avenue is to automate the matching process between the activity types in the activity type and flow structuring template and the activities in the look-ahead schedule using machine learning algorithms.

\section{REFERENCES}

Ballard, G. (1999). "Work structuring." Lean Construction Institute. White paper 5.

Ballard, G., Howell, G., and Zabelle, T. (2001). "Production system design: Work structuring revisited." Lean Construction Institute. White paper 11.

Bertelsen, S., Heinrich, G., and et al. (2007). "Construction Physics." 15th Annual Conference of the International Group for Lean Construction, C. L. Pasquire and P. Tzortzopoulos, eds., East Lansing, Michigan, 13-26.

Bertelsen, S., Koskela, L., Henrich, G., and Rooke, J. (2006). "Critical flow - towards a construction flow theory." 14th Annual Conference of the International Group for Lean Construction, R. Sacks and S. Bertelsen, eds., Santiago, Chile, 31-40.

Darwiche, A., Levitt, R., and Hayes-Roth, B. (1988). "OARPLAN: generating project plans by reasoning about objects, actions, resources." Artificial Intelligence for Engineering, Design, Analysis and Manufacturing, 2(3), 169-181.

Dong, N. (Tony). (2012). "Quick generation of close-to-optimum look-ahead schedules for the finishing phase of complex projects." PhD Dissertation, Stanford University.

Fischer, M. A., and Aalami, F. (1996). "Scheduling with computer-interpretable construction method models." Journal of Construction Engineering and Management, American Society of Civil Engineers, 122(4), 337-347.

Fischer, M., Garcia-Lopez, N. P., and Morkos, R. (2018). "Making each workhour count: Improving the prediction of construction durations and resource allocations." Advanced Computing Strategies for Engineering. EG-ICE 2018. Lecture Notes in Computer Science, vol 10863., I. Smith and B. Domer, eds., Springer, Cham, 273295.

Frandson, A., Berghede, K., and Tommelein, I. D. (2013). "Takt time planning for construction of exterior cladding." 21 st Annual Conference of the International Group for Lean Construction, C. T. Formoso and P. Tzortzopoulos, eds., Fortaleza, Brazil, 527-36.

Frandson, A., and Tommelein, I. D. (2014). "Development of a takt-time plan: A case study." Proc. Construction Research Congress, B. Ashur and J. Irizarry, eds., Reston, VA, 1646-1655.

Garcia-Lopez, N. P. (2017). "An activity and flow-based construction model for managing on-site work.” PhD Dissertation, Stanford University. 
Garcia-Lopez, N. P., and Fischer, M. (2016). "A construction workflow model for analyzing the impact of in-project variability." Proc. Construction Research Congress, ASCE, Reston, VA, 1998-2007.

González, V., Alarcón, L. F., Maturana, S., and Bustamante, J. A. (2011). "Site management of work-in-process buffers to enhance project performance using the Reliable Commitment Model: Case Study.” Journal of Construction Engineering and Management, American Society of Civil Engineers, 137(9), 707-715.

González, V., Alarcón, L. F., and Molenaar, K. (2009). "Multiobjective design of work-inprocess buffer for scheduling repetitive building projects." Automation in Construction, 18(2), 95-108.

Hamzeh, F. R., Ballard, G., and Tommelein, I. D. (2012). "Rethinking lookahead planning to optimize construction workflow." Lean Construction Journal, 2012, 15-34.

Kenley, R., and Seppänen, O. (2009). Location-based management for construction: planning, scheduling and control. Spon Press, Abingdon, Oxon, UK.

Koskela, L. J. (1999). "Management of production in construction: a theoretical view." 7th Annual Conference of the International Group for Lean Construction, Berkeley, CA, 241-252.

Sacks, R. (2016). "What constitutes good production flow in construction?" Construction Management and Economics, 34(9), 641-656.

Tommelein, I. D. (2017). "Collaborative Takt time planning of non-repetitive work." Proc., 25th Annual Conf. of the Int. Group for Lean Construction, K. Walsh, R. Sacks, and I. Brilakis, eds., Herkalion, Greece, 745-752.

Tsao, C. C. Y., Tommelein, I. D., Swanlund, E. S., and Howell, G. A. (2004). "Work structuring to achieve integrated product-process design." Journal of Construction Engineering and Management, 130(6), 780-789. 
Garcia-Lopez, N.P., Fischer, M., and Alarcón, L.F.

Proceedings IGLC-27, July 2019, Dublin, Ireland 